\title{
The Prognostic Significance of NEK2 in Hepatocellular Carcinoma: Evidence from a Meta-Analysis and Retrospective Cohort Study
}

\author{
YuSheng Cheng ${ }^{a, b} \quad$ XiaoLong Chen ${ }^{a, b} \quad$ LinSen Ye $e^{a, b} \quad$ YinCai Zhang ${ }^{a, b} \quad$ Jing Liang ${ }^{c}$ \\ Wei Liu ${ }^{a, b} \quad$ BoXuan Zhou ${ }^{a}$ ShiYang Zheng ${ }^{a}$ Yiming Huang ${ }^{a, b} \quad$ GuiHua Chen ${ }^{a, b}$ \\ YiNan Deng ${ }^{a, b}$ Qi Zhang ${ }^{b, d} \quad$ Yang Yang ${ }^{a, b}$ \\ aDepartment of Hepatic Surgery, The Third Affiliated Hospital of Sun Yat-sen University, Guangzhou, \\ ${ }^{b}$ Guangdong Provincial Key Laboratory of Liver Disease Research, Guangzhou, 'Department of \\ Pathology, Guangdong Key Laboratory of Liver Disease Research, The Third Affiliated Hospital, Sun \\ Yat-sen University, Guangzhou, ${ }^{d}$ Cell-gene Therapy Translational Medicine Research Center, The Third \\ Affiliated Hospital of Sun Yat-sen University, Guangzhou, China
}

\section{Key Words}

NEK2 • Hepatocellular carcinoma • Prognosis • Meta-analysis

\begin{abstract}
Background/Aims: Numerous studies have shown thatNIMA-related kinase2 (NEK2) expression in hepatocellular carcinoma (HCC) tissue is associated with survival and clinicopathological features; however, the evidence remains inconclusive. Thus, we aimed to further explore the prognostic and clinicopathological significance of NEK2 expression in HCC using a two-part study consisting of a retrospective cohort study and a meta-analysis. Methods: In the cohort study, NEK2 expression in 206 HCC samples and adjacent normal liver tissues was detected by immunohistochemistry (IHC). Patients were divided into a high NEK2 expression group and a low NEK2 expression group by the median value of the immunohistochemical scores. The Kaplan-Meier method with the log-rank test was used to analyze survival outcomes in the two groups, and multivariate analysis based on Cox proportional hazard regression models was applied to identify independent prognostic factors. In the meta-analysis, eligible studies were searched in PubMed, EMBASE, Web of Science, and CNKI databases. STATA version 12.0 (Stata Corporation, College Station, TX) was used for statistical analyses. Results: The IHC results of our cohort study showed higher NEK2 expression in HCC tissues compared with adjacent normal liver tissues. Multivariate analysis revealed that high NEK2 expression was an independent risk factor for poor overall survival (OS) [hazard ratio (HR) $=1.763 ; 95 \% \mathrm{Cl}$, 1.060-2.935; $P=0.029$ ] and disease-free survival (DFS) [hazard ratio $(\mathrm{HR})=1.687 ; 95 \% \mathrm{Cl}$,
\end{abstract}

Y.S. Cheng, X. Chen and L. Ye contributed equally to this work.

\begin{tabular}{ll}
\hline Yang Yang, Qi Zhang & Guangdong Provincial Key Laboratory of Liver Disease Research \\
and YiNan Deng & Guangzhou (China); Tel. +86 20 85253106, Fax +86 20 85252276, \\
& E-Mail yysysu@163.com; kee_kee@126.com; dengyinan2010@163.com
\end{tabular}


1.102-2.584; $\mathrm{P}=0.016$ ] in HCC patients. A total of 11 studies with 1,698 patients were enrolled in the meta-analysis, consisting of 10 studies from the database search and our cohort study. The pooled results revealed that high NEK2 expression correlated closely with poor OS among $\mathrm{HCC}$ patients $(\mathrm{HR}=1.47 ; 95 \% \mathrm{Cl}, 1.21-1.80 ; \mathrm{P}<0.01)$, and DFS/recurrence-free survival (RFS) $(\mathrm{HR}=1.92 ; 95 \% \mathrm{Cl}, 1.41-2.63 ; \mathrm{P}<0.01)$. Additionally, our meta-analysis also showed that the proportion of HCC patients with high NEK2 expression was greater in the group with larger tumors $(>5 \mathrm{~cm})$ than in the group with smaller tumors $(\leq 5 \mathrm{~cm})$ [odds ratio $(\mathrm{OR})=2.02 ; 95 \%$ $\mathrm{Cl}, 1.13-3.64 ; \mathrm{P}<0.01)$. Conclusion: Our study demonstrated that high NEK2 expression is a risk factor for poor survival in HCC patients. More prospective, homogeneous, and multiethnic studies are required to validate our findings.

(C) 2018 The Author(s)

Published by S. Karger AG, Basel

\section{Introduction}

Hepatocellular carcinoma (HCC) is the second most common cause of cancer-related death in men and is the sixth most common cause in women worldwide [1]. East Asia and Africa have the highest rates of HCC in the world [2]. Nearly 55\% of all HCC cases in the world occur in China [3]. Moreover, the incidence of HCC is growing worldwide, especially in the United States and Europe [2, 4]. HCC is characterized by fast infiltrating growth, poor differentiation, and early metastasis [5]. Despite substantial improvements in terms of early diagnosis and therapy, HCC patients still have an unfavorable oncological prognosis [6, 7]. Thus, it is essential that the mechanisms responsible for the progression of HCC are further elucidated to enable the development of novel therapeutic targets for HCC.

NIMA-related kinase 2 (NEK2), a member of the NIMA family of serine/threonine kinases located in the centrosome, plays a crucial part in modulating the cell cycle and mitosis [8]. In addition, NEK2 is also involved in B cell development and immunological responses [9], as well as chromatin condensation, spindle assembly checkpoint, microtubule organization and stabilization, kinetochore attachment, and centrosome duplication [10-12]. Greater NEK2 activity could cause chromosomal instability and abnormal chromosomal replication and rearrangement, which are closely linked with the initiation and progression of cancer $[13,14]$. A body of evidence indicates that $N E K 2$ is overexpressed in many malignancies and its expression level inversely correlates with prognosis, including in colorectal cancer, non-Hodgkin lymphoma, multiple myeloma, lung cancer, prostate cancer breast cancer, glioma and pancreatic ductal cancer [15-22]. Moreover, it was reported that elevated NEK2 expression could contribute to drug resistance in multiple myeloma and triple-negative breast cancer [21,23]. These findings indicate that $N E K 2$ is a vital oncogene and may be a potential therapeutic target for cancer treatment.

Recent reports have suggested that NEK2 expression is related to survival in HCC patients; however, the evidence remains inconclusive. Some studies have suggested that NEK2 is overexpressed in HCC tissues compared with adjacent normal tissues and is associated with poor survival [24-31]. In addition, there is evidence showing that elevated NEK2 expression could enhance stem-like properties, drug resistance, and the progression of HCC $[27,28]$. However, a study by Fu et al. reported that HCC patients with high NEK2 expression had better survival [32].

To date, the prognostic and clinicopathological significance of NEK2 in HCC remains controversial. Thus, we conducted a two-part study that comprising a retrospective cohort study and a meta-analysis to further explore the prognostic and clinicopathological significance of NEK2 in HCC patients. 


\section{Cellular Physiology Cell Physiol Biochem 2018;51:2746-2759

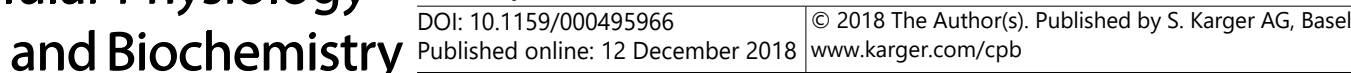 \\ Cheng et al:: The Expression of NEK2 in Hepatocellular Carcinoma}

\section{Materials and Methods}

\section{Study population}

This study was approved by the institutional review board of the Third Affiliated Hospital of Sun Yat-sen University, and written informed consent was obtained from the 206 patients who underwent hepatectomy for HCC between January 2009 and December 2012. These patients were divided into high NEK2 expression and low NEK2 expression groups by median immunohistochemical scores. The diagnosis of hepatocellular carcinoma was histologically confirmed. Patients were excluded if they had preoperative extrahepatic metastasis, other malignancies, or received preoperative anti-cancer therapy. Surgery was performed by several surgeons in accordance with the standard criteria for liver resection in our hospital. Clinical information related to sociodemographic data diagnostic procedures, tumor features, and treatment regimen were collected from medical records. Follow-up information was collected annually from hospital records or direct telephone interviews conducted by trained monitors using structured questionnaires.

\section{Histopathological assessment}

Histopathological evaluation of all surgical specimens was fulfilled based on standard pathological procedures, and a hepatic pathologist reviewed the hematoxylin and eosin (H\&E)-stained histological sections of all patients without any knowledge of the clinical outcomes. The 2010 UICC TNM classification was used to stage the tumors.

\section{Immunohistochemistry (IHC) and IHC evaluation}

IHC was performed to assess the expression of NEK2 in cancerous tissues and matched adjacent noncancerous tissues according to the standard process. Briefly, all surgical samples were formalin-fixed, paraffin embedded, and cut into $4-\mu \mathrm{m}$-thick sections. The sections were deparaffinized, rehydrated, and boiled in a microwave oven with $0.01 \mathrm{~mol} / \mathrm{L}$ sodium citrate buffer ( $\mathrm{pH}$ 6.0) for $10 \mathrm{~min}$ for antigen retrieval. Endogenous peroxidase activity was blocked by hydrogen peroxide (3\%) in PBS and non-specific staining was blocked by BSA. Sections were incubated separately with the primary antibody against NEK2 (ab55550, 1:1, 000 dilution; Abcam, Cambridge, MA, USA) at $4{ }^{\circ} \mathrm{C}$ overnight. After washing, sections were incubated with the secondary antibody (Envision ${ }^{\mathrm{TM}}$ Detection kit; Gene Tech, Shanghai, China) at $37^{\circ} \mathrm{C}$ for $30 \mathrm{~min}$. Finally, immunohistochemical staining was carried out using an EliVision Plus kit (Maixin-Bio, Fuzhou, China); positive staining was visualized with diaminobenzidine (DAB) reagent and counterstained with hematoxylin. IHC was scored in a double-blinded manner by two authors. The German semi-quantitative scoring system, which takes into consideration the staining intensity and percentage of stained cancer cells, was applied to score NEK2 expression [33]. Staining levels were scored as 0 (no staining), 1 (weak staining), 2 (moderate staining), and 3 (strong staining), according to the staining intensity in the tumor cells. The percentage of stained cancer cells in each section was calculated and the sections were scored accordingly $(<10 \%=0 ; 10-25 \%=1 ; 26-50 \%=2 ; 51-75 \%=3 ; 76-100 \%=4)$. The final immunostaining score for each cancerous tissue section was obtained by multiplying the intensity scores with the scores of positively stained cancerous cells, with the scores ranging from 0 to 12 .

\section{Statistical analysis}

The prognostic significance of NEK2 was evaluated by analysis in SPSS 20.0 statistical software (SPSS Inc., Chicago, USA). The relationship between NEK2 expression and patient characteristics was investigated via Pearson's $\chi 2$ test and Spearman's correlation coefficient. The differences in overall survival (OS) and recurrence-free survival (RFS) between the two groups were analyzed using the Kaplan-Meier method with the log-rank test. Univariate analysis was used to establish the potential prognostic factors for OS and DFS, and multivariate analysis for significant factors was performed by Cox proportional hazard regression models. A $p$-value of $<0.05$ indicated a statistically significant difference. 


\section{Cellular Physiology Cell Physiol Biochem 2018;51:2746-2759

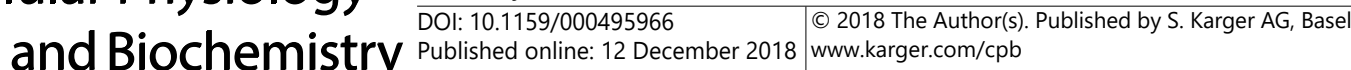

Cheng et al.: The Expression of NEK2 in Hepatocellular Carcinoma

\section{Literature search strategy}

A comprehensive literature search was performed using PubMed, EMBASE, Web of Science, and CNKI for potential eligible articles assessing the prognostic effects of NEK2 in HCC published up until August 11, 2018. The keywords used to perform the searches were the follow: "HCC" or "hepatocellular carcinoma" or "liver cancer" or "liver primary cancer" or "liver primary tumor" or "liver carcinoma"), and ("NEK2" or "NIMA- related kinase 2"). There were no language or regional restrictions in this meta-analysis.

\section{Selection criteria}

All potential articles were screened and selected by two independent authors with the following inclusion criteria: 1) pathologically confirmed HCC; 2) prospective or retrospective cohort study design; and 3 ) reported on the association of NEK2 with survival outcomes or clinicopathological parameters in HCC. The exclusion criteria were as followings: 1) reviews, letters, case reports, meeting abstracts, and metaanalyses; 2) studies investigating other cancers; 3 ) studies without extractable data without accessible full text of articles; and 4) patients not classified into two groups of high NEK2 and low NEK2 expression.

\section{Data collection and quality assessment}

Two investigators independently performed full-text review of eligible articles and extracted the data. The collected data included the name of the first author, publication year, study type, study region, the number of patients in high/low NEK2 expression groups, sex, recruitment period, tumor-nodemetastasis (TNM) stage, detection method, cut-off value, overall survival (OS), disease-free survival (DFS), recurrence-free survival (RFS), and clinicopathological characteristics. Moreover, we treated OS as the primary endpoint because most of the included studies reported the OS. If the hazard ratio (HR) and its 95\% confidence interval (CI) of univariate and multivariate analyses were both reported, only the latter was used since it considers the confounding factors and provides more accuracy. If the HR of survival outcomes was only described using Kaplan-Meier curves, Engauge Digitizer version 4.1 (http://digitizersourceforge. net) was used to extract the survival data, and Tierney's method [35] was used to calculate the estimated HRs and corresponding 95\% CIs. The quality of eligible articles was evaluated by two investigators using the Newcastle-Ottawa Quality Assessment Scale (NOS) [36]. Among the NOS system scores ranging from 0 to 9 , we considered values of 6 and over to represent high-quality articles in this meta-analysis.

\section{Statistical analysis}

STATA version 12.0 (Stata Corporation, College Station, TX, USA) was used to perform the metaanalysis. Synthesized HRs with 95\% CIs were used to describe the association between NEK2 expression and survival in HCC patients, while synthesized odds ratios (ORs) with 95\% CIs were used to assess the association between NEK2 expression and the clinicopathological features of HCC. HR > 1 indicated that NEK2 expression was a risk factor for shorter survival in HCC if the corresponding $95 \%$ CI did not contain 1. Cochran's Q and Higgins $I^{2}$ statistics were used to evaluate the heterogeneity across the studies. We considered $\mathrm{P}<0.05$ and $\mathrm{I}^{2}>50 \%$ to indicate significant heterogeneity, under which conditions the random effects model was applied to synthesize the data. In turn, if there was significant heterogeneity, the fixed effects model was used for pooling the data. To validate the stability of the synthesized results, a sensitivity analysis was carried out by sequentially removing each individual study. Begg's funnel plots and Egger's linear regression tests $[37,38]$ were used to assess publication bias and if the $P$ value was $<0.05$ and the funnel plot was asymmetric, we considered that statistically significant publication bias existed.

\section{Results}

\section{Increased expression of NEK2 in HCC tissues}

NEK2 expression in 206 resected HCC tissue specimens and adjacent non-tumorous liver tissues was analyzed using an IHC staining technique. Immunohistochemical analysis showed that, in all cases, the adjacent non-tumorous liver tissues did not express NEK2. The patients were divided into two arms according to the median values of staining scores: a $N E K 2$-positive expression group $(\mathrm{n}=103)$ and a $N E K 2$-negative expression group $(\mathrm{n}=$ 
103) (Table 1). Furthermore, it was observed from IHC analysis that NEK2 was mainly expressed in the nuclei (Fig. 1).

\section{Correlations between NEK2 expression and clinicopathological parameters \\ For a better understanding of} the clinical value of NEK2 expression in HCC, we further investigated the clinicopathological characteristics among the 206 HCC cases. The expression of NEK2 was significantly higher in cases with large tumors $(\mathrm{P}<0.001)$, and in patients with poor differentiation $(\mathrm{P}=0.002)$ (Table 1). Nevertheless, there were no associations between the expression of $N E K 2$ and the other clinicopathological parameters, including age, sex, tumor number, portal vein thrombosis, TNM stage, HBs antigen, $\alpha$-fetoprotein (AFP) level, and cirrhosis $(\mathrm{P}>0.05)$ (Table 1).

\section{Correlations between NEK2} expression and prognosis

To evaluate the prognostic value of NEK2 expression, we analyzed Kaplan-Meier curves for OS and RFS rates. The 5-year OS and 5-year RFS rates were $46.5 \%$ and $24.7 \%$ for the patients with high NEK2 expression, respectively, and $67.3 \%$ and $54.3 \%$ for patients with no NEK2 expression, respectively. The Kaplan-Meier curves indicated there were significant differences among OS and RFS rates between patients with NKE2-positive expression and those with NEK2-negative expression (Fig. 2). Furthermore, we performed univariate Cox regression analysis to assess the impact of $N E K 2$ expression levels and other common factors on OS and RFS in HCC patients. NEK2 expression ( $\mathrm{P}$ $<0.001)$, differentiation $(\mathrm{P}<0.001)$, portal vein thrombosis $(\mathrm{P}<0.001)$, tumor size $(\mathrm{P}<0.001)$, tumor number $(\mathrm{P}=0.037)$, HBs antigen $(\mathrm{P}=$ 0.004), cirrhosis $(\mathrm{P}=0.034)$ and AFP level $(\mathrm{P}=$ $0.004)$ were determined as crucial risk factors for OS (Table 2). Nevertheless, after multivariate Cox analysis, only NEK2 expression, tumor size, and portal vein thrombosis were found to be independent predictors of OS in patients with
Table 1. Correlation between NIMA-related kinase 2 (NEK2) expression and clinicopathological features. NEK2, NIMAated kinase 2; HBs, antigen, hepatitis B surface antigen;

\begin{tabular}{|c|c|c|c|c|}
\hline \multirow{2}{*}{ Clinical parameters } & \multirow{2}{*}{ Number } & \multicolumn{2}{|c|}{ NEK2 expression } & \multirow{2}{*}{$P$ value } \\
\hline & & Low $(\mathrm{N}=103)$ & $\operatorname{High}(\mathrm{N}=103)$ & \\
\hline \multicolumn{5}{|l|}{ Age (years) } \\
\hline$<55$ & 124 & 58 & 66 & 0.255 \\
\hline$>55$ & 82 & 45 & 37 & \\
\hline \multicolumn{5}{|l|}{ Gender } \\
\hline Male & 180 & 93 & 87 & 0.208 \\
\hline Female & 26 & 10 & 16 & \\
\hline \multicolumn{5}{|l|}{ Tumor size } \\
\hline$\leq 5 \mathrm{~cm}$ & 76 & 55 & 21 & $<0.001$ \\
\hline$>5 \mathrm{~cm}$ & 130 & 48 & 82 & \\
\hline \multicolumn{5}{|l|}{ Tumor number } \\
\hline Single & 145 & 69 & 76 & 0.285 \\
\hline Multiple & 61 & 34 & 27 & \\
\hline \multicolumn{5}{|l|}{ Differentiation } \\
\hline Well/moderate & 155 & 87 & 68 & 0.002 \\
\hline Poorly & 51 & 16 & 35 & \\
\hline \multicolumn{5}{|c|}{ Portal vein thrombosis } \\
\hline Absence & 162 & 89 & 73 & 0.144 \\
\hline Presence & 44 & 14 & 20 & \\
\hline TNM stage & & & & 0.669 \\
\hline I & 135 & 64 & 61 & \\
\hline II/III & 71 & 39 & 42 & \\
\hline \multicolumn{5}{|l|}{ HBs antigen } \\
\hline Negative & 24 & 10 & 14 & 0.385 \\
\hline Positive & 182 & 93 & 89 & \\
\hline \multicolumn{5}{|l|}{ Cirrhosis } \\
\hline Absence & 40 & 18 & 12 & 0.236 \\
\hline Presence & 176 & 85 & 91 & \\
\hline \multicolumn{5}{|l|}{$\operatorname{AFP}(\mathrm{ng} / \mathrm{ml})$} \\
\hline$\leq 400$ & 90 & 47 & 43 & 0.574 \\
\hline$>400$ & 116 & 56 & 60 & \\
\hline
\end{tabular}

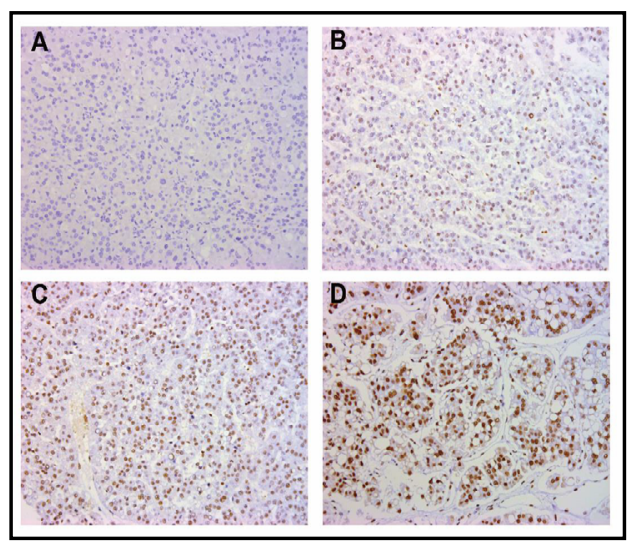

Fig. 1. Immunohistochemical staining of NEK2 in HCC specimens and adjacent noncancerous tissues at $200 \times$ magnification. Negative (-) (A) or weak (+) (B) staining was defined as negative expression; moderate $(++)(\mathrm{C})$ or strong $(+++)$ (D) staining was defined as positive expression. 


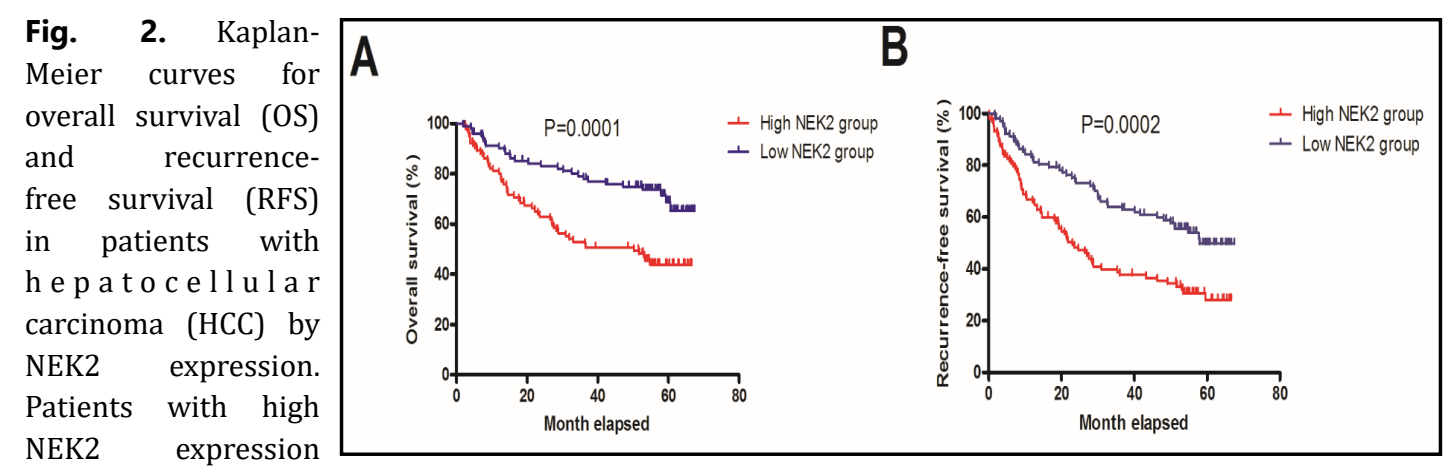
exhibited a shorter OS

(A. $p<0.01$ ) and RFS (B. $p<0.01)$ compared with low NEK2 expression.

HCC (Table 2). With respect to RFS, univariate Cox regression analysis indicated that NEK2 expression (P $<0.001)$, tumor size $(\mathrm{P}<0.001)$, tumor number $(\mathrm{P}=0.037)$, degree of differentiation ( $\mathrm{P}=0.003)$, portal vein thrombosis $(P=0.001)$, and HBs antigen $(\mathrm{P}=0.026)$ were closely associated with RFS (Table 3). However, multivariate Cox analysis suggested that only NEK2 expression and tumor size were independent predictors of RFS in patients with HCC (Table 3).

Study search and study characteristics in meta-analysis

The primary search found 92 potential articles in PubMed, EMBASE, Web of Science, and CNKI. After excluding 7 duplicates, two independent investigators screened the titles and abstracts of the remaining 85 articles, during which 71 articles were excluded as reviews or abstracts, unrelated topics, or nonclinical studies. In the full-text review of the remaining 14 articles, 4 studies were further excluded since the articles did not provide extractable data. Finally, 10 previously published articles and our current cohort study were included in the meta-analysis [25, 30, 32, 39-45]. The details of the literature selection process are presented in Fig. 3.

The basic characteristics of the eligible articles are shown in Table 4. All reports were published between 2016 and 2018. In addition to our cohort study, 9 of the previous studies were conducted in China [25, 32, 39-45], and 1 in Japan [30]. The case numbers in each study ranged between
Table 2. Univariate and multivariate Cox regression analysis for OS (HR hazard ratio, CI confidence interval). OS, overall survival; NEK2, NIMA-related kinase 2; HBs, antigen, hepatitis B surface antigen; AFP, $\alpha$-fetoprotein; HR, hazard ratio; $\mathrm{CI}$, confidence interval; $\mathrm{RFS}$, recurrence-free survival

\begin{tabular}{lllll} 
& \multicolumn{5}{c}{ OS } \\
Variables & HR $(95 \% \mathrm{CI})$ & P-value & HR $(95 \% \mathrm{CI})$ & P-value \\
\hline Age (years) & $0.989(0.968-1.009)$ & 0.282 & & \\
Gender & $1.781(0.821-3.868)$ & 0.144 & & \\
Tumor size & $2.278(1.695-3.061)$ & $<0.001$ & $1.663(1.077-2.567)$ & 0.022 \\
Tumor number & $1.672(1.031-2.711)$ & 0.037 & & \\
Differentiation & $2.628(1.646-4.193)$ & $<0.001$ & & \\
Portal vein thrombosis & $2.009(1.497-2.695)$ & $<0.001$ & $2.395(1.175-4.879)$ & 0.016 \\
TNM stage & $1.068(0.688-1.658)$ & 0.769 & & \\
HBs antigen & $1.910(1.233-2.958)$ & 0.004 & & \\
Cirrhosis & $8.411(1.170-60.462)$ & 0.034 & & \\
AFP & $1.906(1.224-2.966)$ & 0.004 & & \\
NEK2 expression & $2.356(1.493-3.718)$ & $<0.001$ & $1.763(1.060-2.935)$ & 0.029 \\
\hline
\end{tabular}

Table 3. Univariate and multivariate Cox regression analysis for RFS (HR hazard ratio, CI confidence interval). RFS, recurrence-free survival; NEK2, NIMA-related kinase 2; HBs, antigen, hepatitis B surface antigen; AFP, $\alpha$-fetoprotein; HR, hazard ratio; CI, confidence interval; $\mathrm{RFS}$, recurrence-free survival

\begin{tabular}{lllll}
\hline & & \multicolumn{1}{c}{ RFS } & & \\
Variables & HR $(95 \% \mathrm{Cl})$ & P-value & HR $(95 \% \mathrm{CI})$ & P-value \\
\hline Age (years) & $0.994(0.976-1.011)$ & 0.461 & & \\
Gender & $1.627(0.714-3.625)$ & 0.212 & & \\
Tumor size & $1.796(1.416-2.278)$ & $<0.001$ & $1.747(1.233-2.475)$ & 0.002 \\
Tumor number & $1.710(1.366-2.141)$ & $<0.001$ & & \\
Differentiation & $1.765(1.219-2.555)$ & 0.003 & & \\
Portal vein thrombosis & $1.490(1.166-1.903)$ & 0.001 & & \\
TNM stage & $1.270(0.881-1.830)$ & 0.200 & & \\
HBs antigen & $2.170(1.099-4.286)$ & 0.026 & & \\
Cirrhosis & $2.445(0.998-5.991)$ & 0.051 & & \\
AFP & $1.387(0.962-1.999)$ & 0.080 & & \\
NEK2 expression & $1.994(1.374-2.893)$ & $<0.001$ & $1.687(1.102-2.584)$ & 0.016 \\
\hline
\end{tabular}




\section{Cellular Physiology Cell Physiol Biochem 2018;51:2746-2759 and Biochemistry \begin{tabular}{l|l} 
DOI: $10.1159 / 000495966$ & $\begin{array}{l}\text { C } 2018 \text { The Author(s). Published by S. Karger AG, Basel } \\
\text { www.karger.com/cpb }\end{array}$
\end{tabular}

40 and 359. IHC was the most common method used to detect the expression of NEK2, and all patients in the studies included were classified into two groups, i.e., either high or low NEK2 expression. In addition to our cohort study, the correlation between NEK2 expression and OS was investigated in 7 studies $[30,32,39-41,43$, 45], DFS was reported in 1 study [39], and RFS was reported in 3 [30, 41, 45]. In addition to our cohort study, a total of 8 articles analyzed the relationship

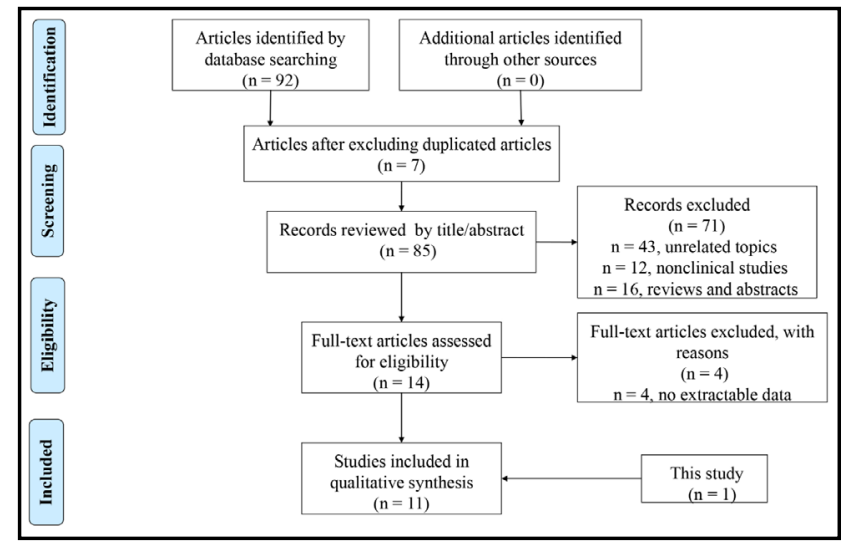

Fig. 3. Flow chart of study selection process. between NEK2 expression and the clinicopathological features, with 7 referring to tumor size $[25,32,39,41,42,44,45], 6$ referring to tumor differentiation $[25,30,32,39,41,45], 5$ referring to tumor number [30, $32,41,42,45], 3$ referring to portal vein thrombosis [25, 27, 30], 7 referring to AFP level $[24,25,28,30-32,45], 5$ referring to HBV status [30, 32, 39, 42, 45], and 5 referring to liver cirrhosis $[32,41,42,44,45]$. The NOS scores of the studies included ranged from 5 to 7 , indicating that the quality of these studies was moderate to high.

\section{Meta-analysis of NEK2 expression and OS in HCC patients}

There were 8 studies that included 1, 542 patients and analyzed the link between NEK2 expression and OS, including 7 previously published studies [30, 32, 39-41, 43, 45] and our cohort study. The fixed model effect was used to synthesize the data, since no significant heterogeneity existed among these studies $\left(\mathrm{I}^{2}=28.3 \%, \mathrm{P}=0.202\right)$. The pooled result showed that high NEK2 expression correlated closely with worse OS in HCC patients (HR $=1.47 ; 95 \%$ CI, 1.21 to 1.80 ; $\mathrm{P}<0.01$ ) (Fig. 4A).

\section{Meta-analysis of NEK2 expression and DFS/RFS in HCC patients}

Among the eligible studies, 4 referred to the correlation between NEK2 expression and DFS/RFS, including 3 previously published studies $[24,27,30]$ and our cohort study. Because of the similarity between DFS and RFS, we merged them for a pooled analysis. Considering that there was no evidence of significant heterogeneity among the 4 studies $\left(\mathrm{I}^{2}=0 \%, \mathrm{p}=\right.$ 0.393), we used the fixed effects model to conduct the meta-analysis. We found that HCC patients with high NEK2 expression had shorter DFS/RFS (HR = 1.92; 95\% CI, 1.41 to 2.63; $\mathrm{P}<0.01$ ). (Fig. 4B).

\section{Meta-analysis of NEK2 expression and clinicopathological features in HCC patients}

To comprehensively explore the roles of NEK2 in HCC, we further investigated the relationships between high expression of $N E K 2$ and the clinicopathological features. The results indicated that the proportion of patients with high expression of $N E K 2$ was greater in the large tumor size group $(>5 \mathrm{~cm})$ than the group with smaller tumors $(\leq 5 \mathrm{~cm})(\mathrm{OR}=$ 2.02; 95\% CI, 1.13-3.64; P < 0.01) (Fig. 5). However, there were no significant differences in the proportion of patients with high expression of $N E K 2$ in a comparison of serum AFP concentrations ( $>400 \mathrm{ng} / \mathrm{mL}$ vs $\leq 400 \mathrm{ng} / \mathrm{mL})(\mathrm{OR}=1.45 ; 95 \% \mathrm{CI}, 0.95-2.20 ; \mathrm{P}<0.01)$ (Fig. 6A), tumor differentiation (well/moderately vs poorly differentiated) (OR $=1.37 ; 95 \%$ $\mathrm{CI}, 0.76-2.47 ; \mathrm{P}=0.12$ ) (Fig. $6 \mathrm{~B})$, tumor number (multiple vs single) $(\mathrm{OR}=1.05 ; 95 \% \mathrm{CI}$, $0.78-1.42 ; \mathrm{P}=0.14$ ) (Fig. $6 \mathrm{C}$ ), portal vein thrombosis (present vs absent) (OR $=1.88 ; 95 \% \mathrm{CI}$, $0.78-4.51 ; \mathrm{P}=0.12$ ) (Fig. 6D), liver cirrhosis (present vs absent) $(\mathrm{OR}=1.16$; $95 \% \mathrm{CI}, 0.87-$ 1.54; $\mathrm{P}=0.07$ ) (Fig. $6 \mathrm{E}$ ), or HBV status (positive vs negative) (OR $=0.85 ; 95 \% \mathrm{CI}, 0.59-1.21$; $\mathrm{P}=0.11$ ) (Fig. 6F). 


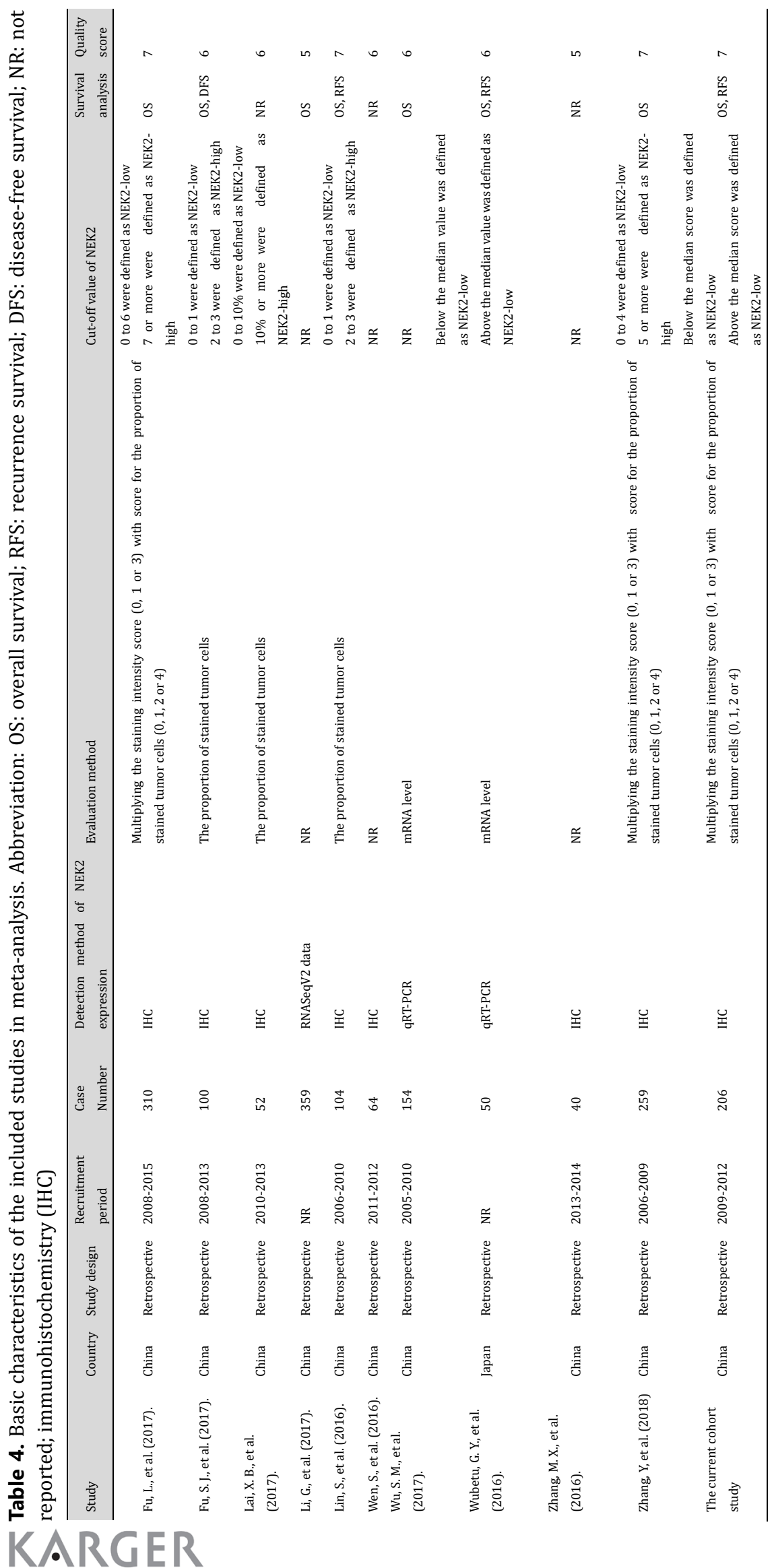




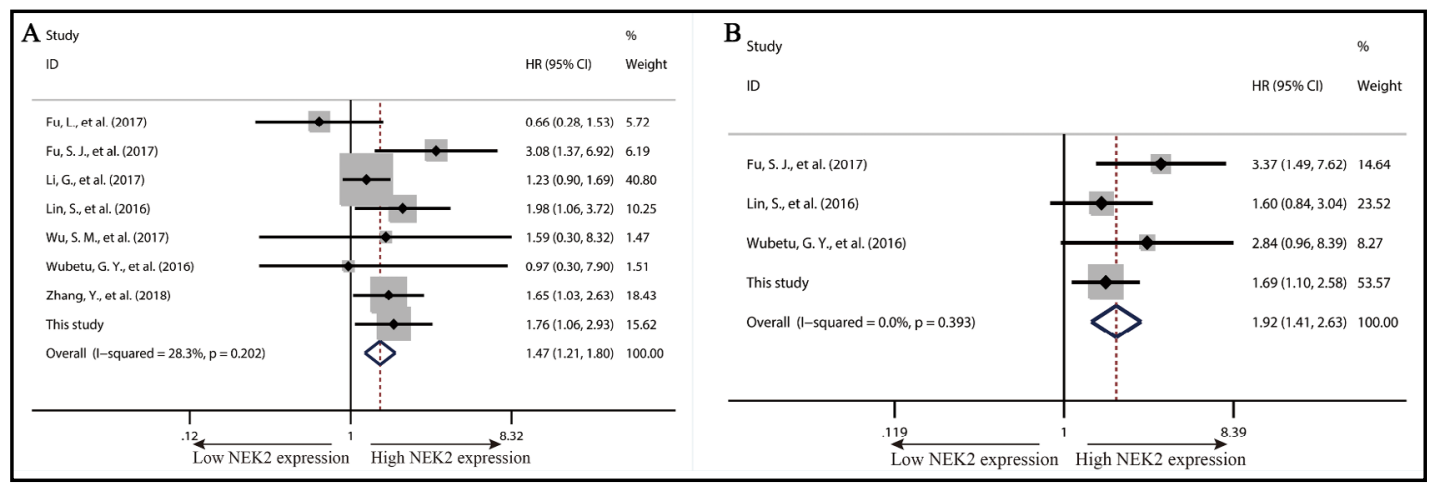

Fig. 4. Forest plots for pooled HR with $95 \%$ CI for the associations between NEK2 expression with overall survival and disease-free survival.

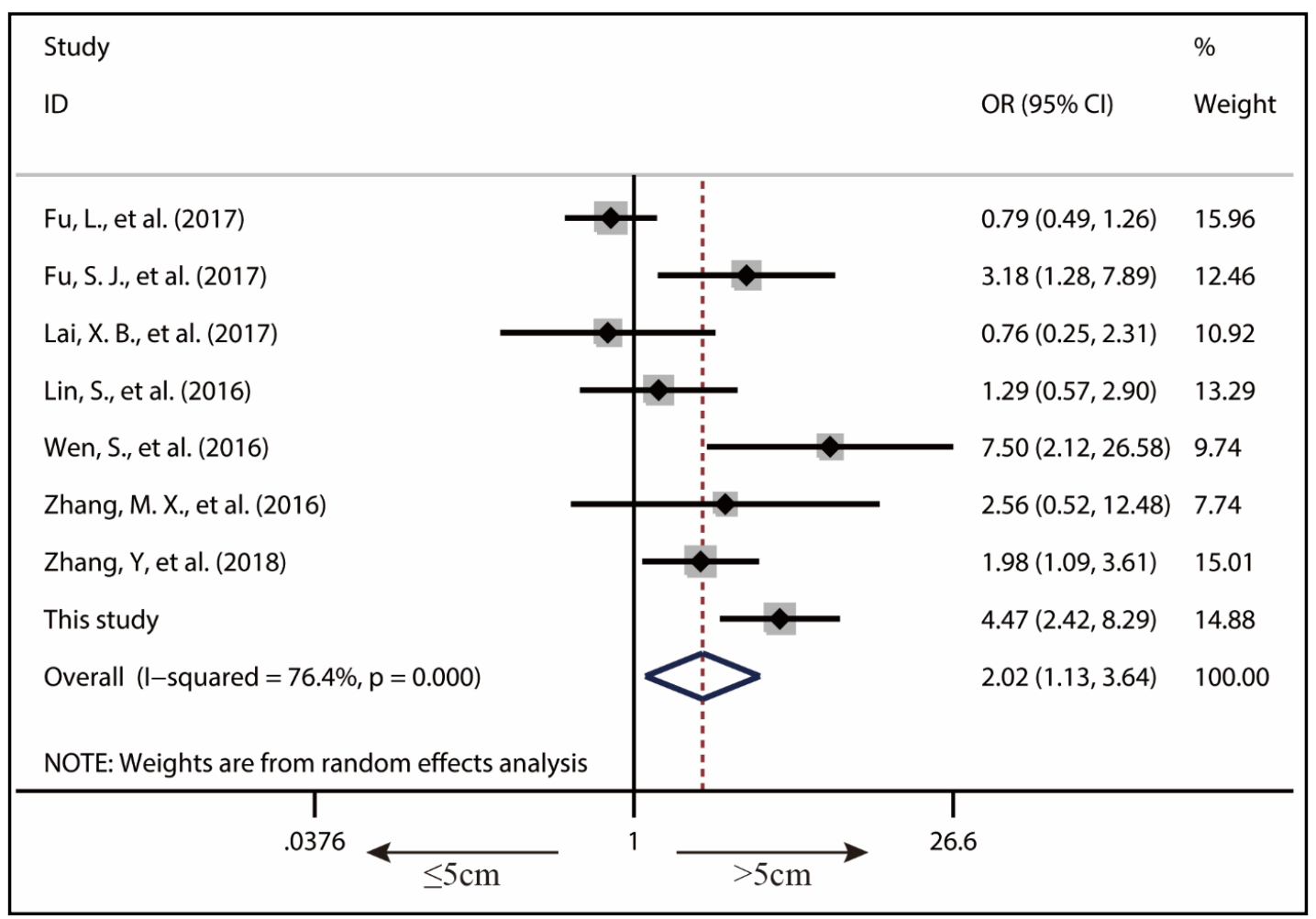

Fig. 5. Forest plots for pooled OR with $95 \%$ CI for the proportion of HCC patients with high NEK2 expression and the comparison of tumor size.

\section{Sensitivity analysis}

To evaluate the stability of the synthesized HR for OS, a sensitivity analysis was performed by sequentially removing each individual study. The result showed that the synthesized HR did not alter substantially with the removal of any individual study, indicating that the pooled HR of OS was stable and reliable (Fig. 7A).

\section{Publication bias}

Begg's funnel plots and Egger's linear regression tests were used to assess publication bias of the synthesized HR for OS. The results showed that Begg's funnel plot was symmetrical (Fig. 7B), and the $\mathrm{p}$ value for Egger's regression asymmetry test was 0.784 , suggesting that there was no significant publication bias.

\section{KARGER}




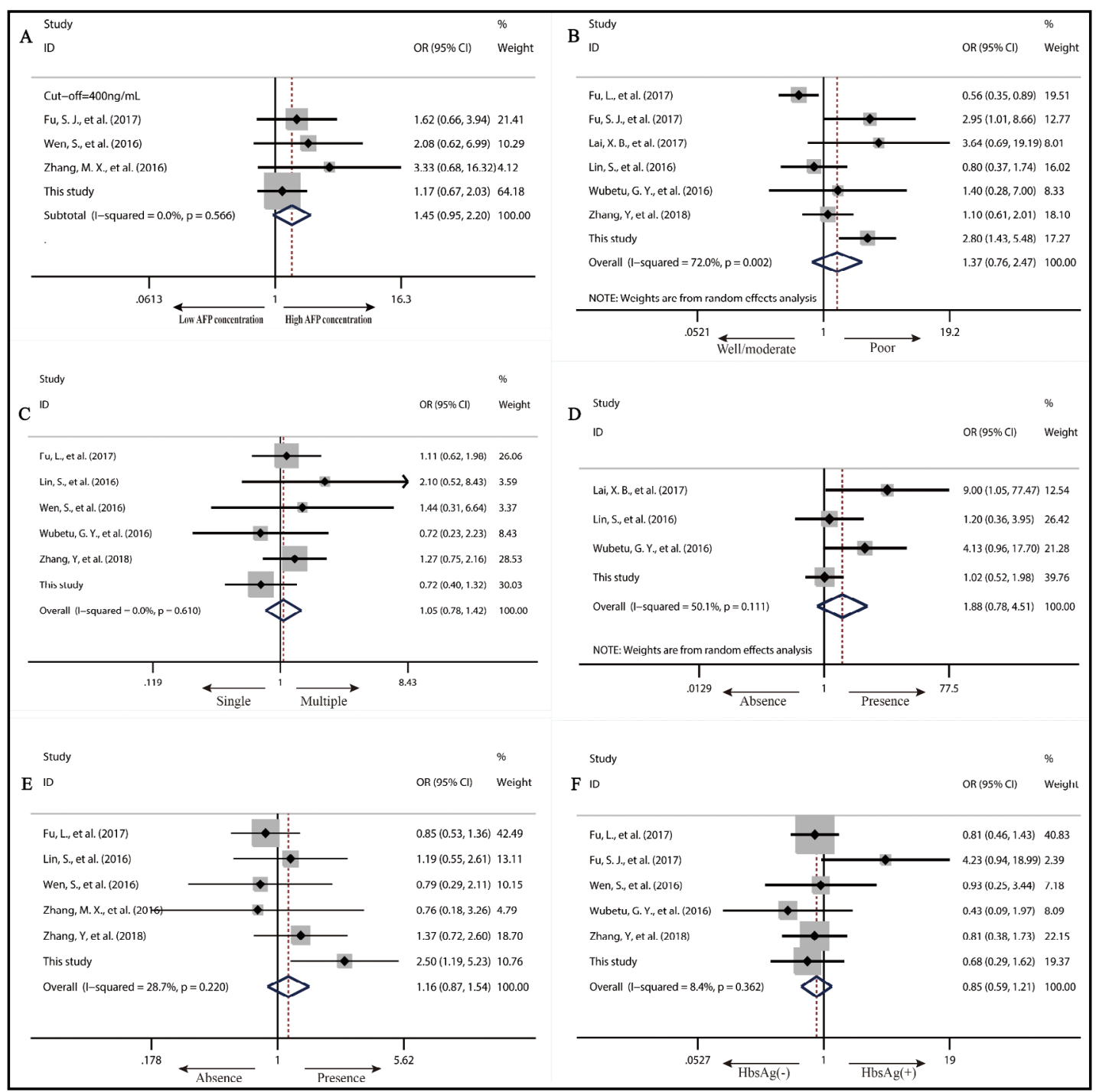

Fig. 6. Forest plots for pooled OR with $95 \%$ CI for the proportion of HCC patients with high NEK2 expression in comparisons of serum AFP concentration ( $>400 \mathrm{ng} / \mathrm{mL}$ vs $\leq 400 \mathrm{ng} / \mathrm{mL}$ ) (A), tumor differentiation (well/ moderately vs poorly differentiated) (B), tumor number (multiple vs single) (C), portal vein thrombosis (present vs absent) (D), liver cirrhosis (present vs absent) (E), and HBV status (positive vs negative).

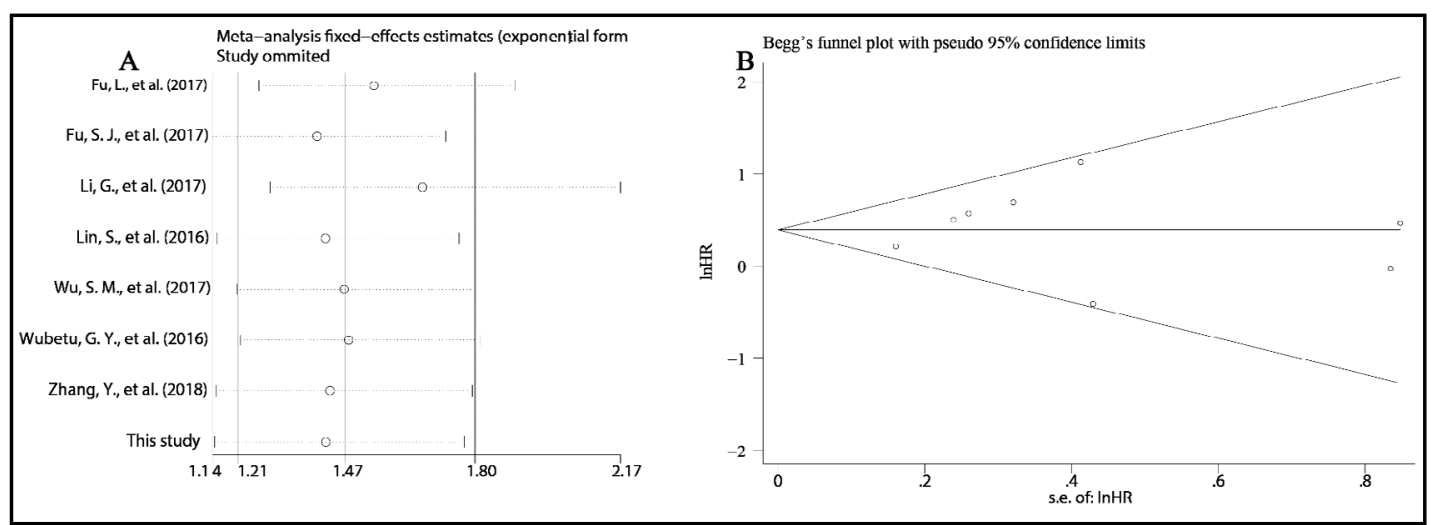

Fig. 7. Sensitivity analysis of pooled HR for overall survival (OS) (A); funnel plots of Begg's test estimating publication bias of pooled HR for OS (B). s.e., standard error. 


\section{Cellular Physiology Cell Physiol Biochem 2018;51:2746-2759 and Biochemistry Published online: T2 December $2018 \begin{aligned} & \text { DOI: } 2018 \text { The Author(s). Published by S. Karger AG, Basel } \\ & \text { www.karger.com/cpb }\end{aligned}$ \\ Cheng et al:: The Expression of NEK2 in Hepatocellular Carcinoma}

\section{Discussion}

Comprehensive analyses of the molecular mechanisms underlying the initiation and progression of HCC play a role in improving the rates of early diagnosis and individualized therapy. This two-step study focused on thoroughly exploring the prognostic and clinicopathological significance of NEK2 expression and reviewed its relevant molecular mechanisms in HCC. Consistent with the majority of previous studies [24-31], our current cohort study also showed that NEK2 was overexpressed in HCC tissues compared with adjacent normal tissues, and high NEK2 expression correlated positively with larger tumor size, portal vein invasion, and poor tumor differentiation. However, the meta-analysis showed that high NEK2 expression was only related to larger tumor size and not to the other clinicopathological features. With respect to survival outcomes, both our current cohort study and the meta-analysis confirmed that there were close correlations between high NEK2 expression and shorter OS and DFS/RFS.

Numerous researchers have explored the underlying mechanisms of the prognostic significance of NEK2 in HCC patients. A study by Zhang et al., which investigated the association between NEK2 expression and HCC initiation and progression for the first time, revealed that $N E K 2$ overexpression could promote the proliferation and survival of HepG2 cells by activating the mitogen activated protein kinase (MAPK) signaling pathway. Aberrant activation of Wnt/ $\beta$-catenin signaling pathway plays an important role in sustaining cancer cell stemness [46]. Consistently, Lin et al. reported that the overexpression of NEK2 could enhance self-renewal properties in HCC by activating the Wnt/ $\beta$-catenin pathway [27]. Moreover, a study by Lai et al. suggested that the overexpression of NEK2 could induce cell cycle progression and promote proliferation via the activation of $\mathrm{Wnt} / \beta$-catenin signaling pathway [25]. ABCG2, a member of ABC transporter family, is a vital element of multi-drug resistance system in cancer [47] and Lin et al. demonstrated that NEK2 plays a crucial role in chemotherapeutic resistance by regulating the expression of ABCG2 in HCC cells [27]. Similarly, a study by $\mathrm{Wu}$ et al. demonstrated that $N K E 2$ overexpression can induce the resistance of hepatoma J7 or Hep3B cells to chemotherapy via upregulation of $A B C C 10$ and the expression of Twist [29]. Additionally, abnormal activation of the p-AKT/NF- $\kappa B$ signaling pathway and matrix metalloproteinase- 2 was found to be involved in NEK2 overexpressioninduced migration, invasion, and angiogenesis in HCC [26, 29].

To our knowledge, this two-part study is the first meta-analysis to thoroughly evaluate the prognostic and clinicopathological significance of NEK2 expression in HCC. However, it did have several limitations. First, all eligible studies in the meta-analysis were of a retrospective nature, which might have introduced a degree of bias. Second, although no statistically significant heterogeneity was found during the meta-analysis of OS and DFS/ RFS, inconsistencies among the treatments, methods of testing for NEK2 expression, cutoff values for high $N E K 2$ expression, and recruitment period might also have introduced a degree of bias and heterogeneity and thus might have influenced the robustness of our pooled results. Third, in some studies, the HRs for OS were calculated by univariate analysis or manually calculated from the Kaplan-Meier curve, which did not exclude the impact of key confounding factors on survival, and thus bias and heterogeneity might have occurred to a degree. Fourth, among the 11 studies included in the meta-analysis, 10 were from China and only 1 study was from Japan, but none focused on Caucasian and African populations. Therefore, future work is required to explore the prognostic value of $N E K 2$ expression in Caucasian and African populations with HCC.

\section{Conclusion}

Our study demonstrated that high expression of NEK2 is a risk factor for poor survival in HCC patients. More prospective, homogeneous, and multi-ethnic studies are required to validate our current findings. 


\section{Cellular Physiology Cell Physiol Biochem 2018;51:2746-2759 and Biochemistry Published \begin{tabular}{l|l} 
DOI: 10.1159/000495966 & $\begin{array}{l}\text { (c) } 2018 \text { The Author(s). Published by S. Karger AG, Basel } \\
\text { www.karger.com/cpb }\end{array}$
\end{tabular}}

Cheng et al.: The Expression of NEK2 in Hepatocellular Carcinoma

\section{Acknowledgements}

This work was supported by the grants from National Natural Science Foundation of China (81770648, 81702393, 81570593, 81370555, 81702409), National Key R\&D Program of China (2017YFA0104304), Guangdong Natural Science Foundation (2017A030310373, 2017A030310062, 2018A030313043), Sci-tech Research Development Program of Guangdong province (2017A020215023), Guangzhou Science and Technology Plan Project \& Guangzhou Clinical Medical Research and Transformation Center Pilot Construction Projct (2014Y2-00544), Young Teacher Development Program of Sun Yat-Sen University (17ykpy57), Sun Yat-sen University Young Teacher Training Project (17ykpy47), Medical Scientific Research Foundation of Guangdong Province, China (A2017336), and 3rd Affiliated Hospital of Sun Yat-Sen University, Clinical Research Program (QHJH201801).

\section{Disclosure Statement}

The authors declare that there were no conflicts of interest.

\section{References}

1 Torre LA, Bray F, Siegel RL, Ferlay J, Lortet-Tieulent J, Jemal A: Global cancer statistics, 2012. CA Cancer J Clin 2015;65:87-108.

- Forner A, Llovet JM, Bruix J: Hepatocellular carcinoma. Lancet 2012;379:1245-1255.

3 Yuen MF, Hou JL, Chutaputti A: Hepatocellular carcinoma in the Asia pacific region. J Gastroenterol Hepatol 2009;24:346-353.

4 Jemal A, Simard EP, Dorell C, Noone AM, Markowitz LE, Kohler B, Eheman C, Saraiya M, Bandi P, Saslow D, Cronin KA, Watson M, Schiffman M, Henley SJ, Schymura MJ, Anderson RN, Yankey D, Edwards BK: Annual Report to the Nation on the Status of Cancer, 1975-2009, featuring the burden and trends in human papillomavirus(HPV)-associated cancers and HPV vaccination coverage levels. J Natl Cancer Inst 2013;105:175-201.

5 Lazarevich NL, Cheremnova OA, Varga EV, Ovchinnikov DA, Kudrjavtseva EI, Morozova OV, Fleishman DI, Engelhardt NV, Duncan SA: Progression of HCC in mice is associated with a downregulation in the expression of hepatocyte nuclear factors. Hepatology 2004;39:1038-1047.

-6 Liu Z, Tu K, Wang Y, Yao B, Li Q, Wang L, Dou C, Liu Q, Zheng X: Hypoxia Accelerates Aggressiveness of Hepatocellular Carcinoma Cells Involving Oxidative Stress, Epithelial-Mesenchymal Transition and NonCanonical Hedgehog Signaling. Cell Physiol Biochem 2017;44:1856-1868.

7 Zheng J, Cai J, Li H, Zeng K, He L, Fu H, Zhang J, Chen L, Yao J, Zhang Y, Yang Y: Neutrophil to Lymphocyte Ratio and Platelet to Lymphocyte Ratio as Prognostic Predictors for Hepatocellular Carcinoma Patients with Various Treatments: a Meta-Analysis and Systematic Review. Cell Physiol Biochem 2017;44:967-981. Fry AM, Bayliss R, Roig J: Mitotic Regulation by NEK Kinase Networks. Front Cell Dev Biol 2017;5:102.

9 Gu Z, Zhou W, Huang J, Yang Y, Wendlandt E, Xu H, He X, Tricot G, Zhan F: Nek2 is a novel regulator of B cell development and immunological response. Biomed Res Int 2014;2014:621082.

$>10$ Fry AM: The Nek2 protein kinase: a novel regulator of centrosome structure. Oncogene 2002;21:61846194.

11 Fry AM, O’Regan L, Sabir SR, Bayliss R: Cell cycle regulation by the NEK family of protein kinases. J Cell Sci 2012;125:4423-4433.

12 Wang G, Jiang Q Zhang C: The role of mitotic kinases in coupling the centrosome cycle with the assembly of the mitotic spindle. J Cell Sci 2014;127:4111-4122.

13 Cappello P, Blaser H, Gorrini C, Lin DC, Elia AJ, Wakeham A, Haider S, Boutros PC, Mason JM, Miller NA, Youngson B, Done SJ, Mak TW: Role of Nek2 on centrosome duplication and aneuploidy in breast cancer cells. Oncogene 2014;33:2375-2384.

14 Hayward DG, Fry AM: Nek2 kinase in chromosome instability and cancer. Cancer Lett 2006;237:155-166. 


\section{Cellular Physiology Cell Physiol Biochem 2018;51:2746-2759

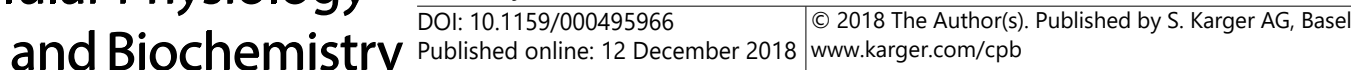

Cheng et al.: The Expression of NEK2 in Hepatocellular Carcinoma

15 Gu Z, Xia J, Xu H, Frech I, Tricot G, Zhan F: NEK2 Promotes Aerobic Glycolysis in Multiple Myeloma Through Regulating Splicing of Pyruvate Kinase. J Hematol Oncol 2017;10:17.

16 Lu L, Zhai X, Yuan R: Clinical significance and prognostic value of Nek2 protein expression in colon cancer. Int J Clin Exp Pathol 2015;8:15467-15473.

17 Ning Z, Wang A, Liang J, Liu J, Zhou T, Yan Q Wang Z: Abnormal expression of Nek2 in pancreatic ductal adenocarcinoma: a novel marker for prognosis. Int J Clin Exp Pathol 2014;7:2462-2469.

-18 Nuncia-Cantarero M, Martinez-Canales S, Andres-Pretel F, Santpere G, Ocana A, Galan-Moya EM: Functional transcriptomic annotation and protein-protein interaction network analysis identify NEK2, BIRC5, and TOP2A as potential targets in obese patients with luminal A breast cancer. Breast Cancer Res Treat 2018;10.1007/s10549-017-4652-3.

19 Shi YX, Yin JY, Shen Y, Zhang W, Zhou HH, Liu ZQ: Genome-scale analysis identifies NEK2, DLGAP5 and ECT2 as promising diagnostic and prognostic biomarkers in human lung cancer. Sci Rep 2017;7:8072.

20 Zeng YR, Han ZD, Wang C, Cai C, Huang YQ Luo HW, Liu ZZ, Zhuo YJ, Dai QS, Zhao HB, Liang YX, Zhong WD: Overexpression of NIMA-related kinase 2 is associated with progression and poor prognosis of prostate cancer. BMC Urol 2015;15:90.

21 Zhou W, Yang Y, Xia J, Wang H, Salama ME, Xiong W, Xu H, Shetty S, Chen T, Zeng Z, Shi L, Zangari M, Miles R, Bearss D, Tricot G, Zhan F: NEK2 induces drug resistance mainly through activation of efflux drug pumps and is associated with poor prognosis in myeloma and other cancers. Cancer Cell 2013;23:48-62.

-22 Liu H, Liu B, Hou X, Pang B, Guo P, Jiang W, Ding Q Zhang R, Xin T, Guo H, Xu S, Pang Q: Overexpression of NIMA-related kinase 2 is associated with poor prognoses in malignant glioma. J Neurooncol 2017;132:409417.

23 Lee J, Gollahon L: Nek2-targeted ASO or siRNA pretreatment enhances anticancer drug sensitivity in triplenegative breast cancer cells. Int J Oncol 2013;42:839-847.

-24 Fu SJ, Chen J, Ji F, Ju WQ, Zhao Q, Chen MG, Guo ZY, Wu LW, Ma Y, Wang DP, Zhu XF, He XS: MiR-486-5p negatively regulates oncogenic NEK2 in hepatocellular carcinoma. Oncotarget 2017;8:52948-52959.

-25 Lai XB, Nie YQ, Huang HL, Li YF, Cao CY, Yang H, Shen B, Feng ZQ: NIMA-related kinase 2 regulates hepatocellular carcinoma cell growth and proliferation. Oncol Lett 2017;13:1587-1594.

26 Li G, Zhong Y, Shen Q, Zhou Y, Deng X, Li C, Chen J, Zhou Y, He M: NEK2 serves as a prognostic biomarker for hepatocellular carcinoma. Int J Oncol 2017;50:405-413.

27 Lin S, Zhou S, Jiang S, Liu X, Wang Y, Zheng X, Zhou H, Li X, Cai X: NEK2 regulates stem-like properties and predicts poor prognosis in hepatocellular carcinoma. Oncol Rep 2016;36:853-862.

28 Wen S, Liu Y, Yang M, Yang K, Huang J, Feng D: Increased NEK2 in hepatocellular carcinoma promotes cancer progression and drug resistance by promoting PP1/Akt and Wnt activation. Oncol Rep 2016;36:2193-2199.

29 Wu SM, Lin SL, Lee KY, Chuang HC, Feng PH, Cheng WL, Liao CJ, Chi HC, Lin YH, Tsai CY, Chen WJ, Yeh CT, Lin KH: Hepatoma cell functions modulated by NEK2 are associated with liver cancer progression. Int J Cancer 2017;140:1581-1596.

-30 Wubetu GY, Morine Y, Teraoku H, Yoshikawa M, Ishikawa D, Yamada S, Ikemoto T, Saito YU, Imura S, Shimada M: High NEK2 Expression Is a Predictor of Tumor Recurrence in Hepatocellular Carcinoma Patients After Hepatectomy. Anticancer Res 2016;36:757-762.

-31 Zhang MX, Xu XM, Zhang P, Han NN, Deng JJ, Yu TT, Gan YY, He XQ, Long ZX: Effect of silencing NEK2 on biological behaviors of HepG2 in human hepatoma cells and MAPK signal pathway. Tumour Biol 2016;37:2023-2035.

32 Fu L, Liu S, Wang H, Ma Y, Li L, He X, Mou X, Tong X, Hu Z, Ru G: Low expression of NEK2 is associated with hepatocellular carcinoma progression and poor prognosis. Cancer Biomark 2017;20:101-106.

33 Mao Z, Sun J, Feng B, Ma J, Zang L, Dong F, Zhang D, Zheng M: The metastasis suppressor, N-myc downregulated gene 1 (NDRG1), is a prognostic biomarker for human colorectal cancer. PLoS One 2013;8:e68206.

34 Knobloch K, Yoon U, Vogt PM: Preferred reporting items for systematic reviews and meta-analyses (PRISMA) statement and publication bias. J Craniomaxillofac Surg 2011;39:91-92.

35 Tierney JF, Stewart LA, Ghersi D, Burdett S, Sydes MR: Practical methods for incorporating summary timeto-event data into meta-analysis. Trials 2007;8:16.

-36 Stang A: Critical evaluation of the Newcastle-Ottawa scale for the assessment of the quality of nonrandomized studies in meta-analyses. Eur J Epidemiol 2010;25:603-605. 


\section{Cellular Physiology Cell Physiol Biochem 2018;51:2746-2759 and Biochemistry DOl: 10.1159/000495966 2018 O 2018 The Author(s). Published by S. Karger AG, Basel

-37 Begg CB, Mazumdar M: Operating characteristics of a rank correlation test for publication bias. Biometrics 1994;50:1088-1101.

-38 Egger M, Davey Smith G, Schneider M, Minder C: Bias in meta-analysis detected by a simple, graphical test. BMJ 1997;315:629-634.

-39 Fu SJ, Chen J, Ji F, Ju WQ, Zhao Q, Chen MG, Guo ZY, Wu LW, Ma Y, Wang DP: MiR-486-5p negatively regulates oncogenic NEK2 in hepatocellular carcinoma. Oncotarget 2017;8:52948-52959.

-40 Li G, Zhong Y, Shen Q, Zhou Y, Deng X, Li C, Chen J, Zhou Y, He M: NEK2 serves as a prognostic biomarker for hepatocellular carcinoma. International Journal of Oncology 2017;50:405-413.

-41 Lin S, Zhou S, Jiang S, Liu X, Wang Y, Zheng X, Zhou H, Li X, Cai X: NEK2 regulates stem-like properties and predicts poor prognosis in hepatocellular carcinoma. Oncology Reports 2016;36:853.

42 Wen S, Liu Y, Yang M, Yang K, Huang J, Feng D: Increased NEK2 in hepatocellular carcinoma promotes cancer progression and drug resistance by promoting PP1/Akt and Wnt activation. Oncology Reports 2016;36:2193.

43 Wu SM, Lin SL, Lee KY, Chuang HC, Feng PH, Cheng WL, Liao CJ, Chi HC, Lin YH, Tsai CY: Hepatoma cell functions modulated by NEK2 are associated with liver cancer progression. Int J Cancer. 2017;140:15811596.

44 Zhang MX, Xu XM, Zhang P, Han NN, Deng JJ, Yu TT, Gan YY, He XQ, Long ZX: Effect of silencing NEK2 on biological behaviors of HepG2 in human hepatoma cells and MAPK signal pathway. Tumor Biology 2016;37:2023-2035.

45 Zhang Y, Wang W, Wang Y, Huang X, Zhang Z, Chen B, Xie W, Li S, Shen S, Peng B: NEK2 promotes hepatocellular carcinoma migration and invasion through modulation of the epithelial-mesenchymal transition. Oncol Rep 2018;39:1023-1033.

46 Fodde R, Brabletz T: Wnt/beta-catenin signaling in cancer stemness and malignant behavior. Curr Opin Cell Biol 2007;19:150-158.

$\checkmark 47$ Ho RH, Kim RB: Transporters and drug therapy: implications for drug disposition and disease. Clin Pharmacol Ther 2005;78:260-277. 